\title{
Knockdown of spindle pole body component 25 homolog inhibits cell proliferation and cycle progression in prostate cancer
}

\author{
FEILUN CUI $^{1}$, JIANPENG HU ${ }^{1}$, YU FAN $^{2}$, JIAN TAN ${ }^{1}$ and HUAMING TANG ${ }^{1}$ \\ Departments of ${ }^{1}$ Urology and ${ }^{2}$ Oncology, The Affiliated People's Hospital of Jiangsu University, \\ Zhenjiang, Jiangsu 212000, P.R. China
}

Received August 11, 2017; Accepted December 22, 2017

DOI: $10.3892 / \mathrm{ol} .2018 .8003$

\begin{abstract}
Prostate cancer (PCa) is the most frequently diagnosed type of cancer in Chinese males. Cell-cycle aberration is a hallmark of cancer. Spindle pole body component 25 homolog (SPC25), a component of the Ndc80 complex, serves an important role in regulating mitotic chromosome segregation. However, the functional roles of SPC25 in PCa remain poorly understood. To the best of our knowledge, the present study was the first to demonstrate that SPC25 is significantly upregulated in PCa. In order to investigate the molecular roles of SPC25, a loss of function assay was performed, revealing that SPC25 knockdown inhibited cell proliferation, and induced a decrease in the number of cells in the $\mathrm{S}$ phase and an increase in the number of cells in the G2/M phase. Furthermore, SPC25 knockdown promoted the apoptosis of PCa cells. Additionally, bioinformatics analysis revealed multiple functional roles of SPC25 in regulating cell proliferation, apoptosis, invasion, transforming growth factor- $\beta$ signaling and the SUMOylation pathway in PCa. The present study also evaluated the potential prognostic value of SPC25 using The Cancer Genome Atlas RNA-seq data and demonstrated that SPC25 was upregulated in late stage PCa. Kaplan-Meier analysis demonstrated that lower SPC25 expression was associated with an improved survival rate in patients with $\mathrm{PCa}$. Taken together, these results suggested that SPC25 serves an oncogenic role in PCa and may act as a novel diagnostic and therapeutic target for $\mathrm{PCa}$.
\end{abstract}

Correspondence to: Dr Jianpeng Hu, Department of Urology, The Affiliated People's Hospital of Jiangsu University, 8 Dianli Road, Zhenjiang, Jiangsu 212000, P.R. China

E-mail: 249056614@qq.com

Professor Yu Fan, Department of Oncology, The Affiliated People's Hospital of Jiangsu University, 8 Dianli Road, Zhenjiang, Jiangsu 212000, P.R. China

E-mail: yuf36@sina.com

Key words: prostate cancer, spindle pole body component 25 homolog, prognosis, proliferation, cell cycle

\section{Introduction}

One of the common hallmarks of cancer is abnormal mitosis (1). Kinetochores are the key complexes that regulate mitotic chromosome segregation by generating physical connections between chromosomes and spindle microtubule polymers $(2,3)$. The Ndc80 complex, a hetero-tetramer protein complex of Ndc80, Nuf2, Spc24 and Spc25, is at the core of the kinetochore and is the key kinetochore coupler (4). Recent studies have demonstrated that abnormal expression of the Ndc80 complex is involved in the progression of human cancer (5-7). For example, NDC80 (7) and NUF2 (6) were reported as oncogenes in colon cancer and osteosarcoma. SPC25, together with SPC24, binds the kinetochore at one end of the Ndc80 complex (8). However, the functional roles of SPC25 in cancer remain unknown.

In the past decade, prostate cancer (PCa) is the most frequently diagnosed type of cancer in Chinese males (9). Over the past three decades, certain genes, including androgen receptor $(10)$, speckle-type POZ protein $(11,12)$, motor neuron and pancreas homeobox $1(13,14)$, were identified as key regulators in $\mathrm{PCa}$. As a result, the survival rate of patients with localized PCa has been improved owing to surgery and radiotherapy (15). However, the molecular mechanisms underlying PCa progression remain poorly understood. Therefore, the identification of novel regulators as diagnostic and therapeutic strategies is urgently required.

The present study investigated the expression of SPC25 in PCa tissues using The Cancer Genome Atlas, and investigated the potential roles of SPC25 in regulating cell proliferation, cell cycle, cell migration and apoptosis. The present study may provide useful information for the identification of novel therapeutic and prognostic targets for $\mathrm{PCa}$.

\section{Materials and methods}

Patients and clinicopathological data. The detailed SPC25 expression data of 490 patients with PCa were downloaded from The Cancer Genome Atlas (TCGA, https://tcga-data. nci.nih.gov/tcga/) database by using the Firebrowse dataset (http://firebrowse.org/) (16,17). The RNA expression data (level 3) were generated from the HiSeq 2000 sequencing platform (Illumina Inc., San Diego, CA, USA) by RNASeqV2 post-processing pipelines and were presented as RNA-Seq by Expectation-Maximization normalized count data. Patient 
clinical features, including age at diagnosis, days to last follow-up, pathological tumor $(\mathrm{T})$ stage and node $(\mathrm{N})$ stage, were retrospectively obtained from patient records. All the patients were staged using the 2009 Tumor-Node-Metastasis (TNM) classification of the American Joint Committee on Cancer/International Union Against Cancer (18). The Gleason grading system was also used to evaluate the prognosis of men with prostate cancer using samples from a prostate biopsy. The Gleason scores range from 2 to 10, with higher number indicating greater risks and higher mortality (19). In order to further investigate the prognostic value of SPC25 in $\mathrm{PCa}$, the overall survival rates of patients with high or low SPC25 expression were assessed using the Kaplan-Meier method by using GSE21032 dataset, which was reported by Taylor et al. The upper 75\% SPC25 mRNA expression in all PCa tissues was used as the cut-off point to divide all cases into high ( $\mathrm{n}=36, \geq 75 \%$ SPC25 expression), and low $(\mathrm{n}=105$, $<75 \%$ SPC25 expression), groups and clinicopathological characteristics, including the Gleason score.

Lentiviral constructs and transfections. A total of $6 \mu \mathrm{g}$ SureSilencing short hairpin RNA (shRNA) plasmids (Qiagen $\mathrm{GmbH}$ ) were used against SPC25 to knockdown SPC25 expression levels using standard molecular techniques. The SPC25 shRNA sequence was as follows: 3'-CCGGC CATCAAAGCATTTGCAGAAACTCGAGTTTCTGCAAA TGCTTTGATGGTTTTTG-5', which were purchased from Shanghai GenePharma Co., Ltd. (Shanghai, China). 293T cells were infected with the recombinant lentiviral vectors using Lipofectamine ${ }^{\circledR} 2000$ (Thermo Fisher Scientific, Inc., Waltham, MA, USA) to generate stably transfected cells. A total of $4 \mathrm{~h}$ after transfection, the Opti-MEM was changed to RPMI-1640 medium containing $10 \%$ fetal bovine serum (GE Healthcare Life Sciences, Little Chalfont, UK), 100 U/ml penicillin and $100 \mu \mathrm{g} / \mathrm{ml}$ streptomycin, and were cultured at $37^{\circ} \mathrm{C}$ in $5 \% \mathrm{CO}_{2}$. After $24 \mathrm{~h}$, concentrated lentiviruses were collected. Opti-modified Eagle's medium (Opti-MEM) which was ideal for use during cationic lipid transfections especially Lipofectamine 2000 transfection reagents was purchased from Thermo Fisher Scientific, Inc (cat. no. 31985062). Concentrated lentiviruses were transfected at a multiplicity of infection (MOI) of 40 in serum-free RPMI-1640 medium. The supernatant was replaced with complete culture medium (RPMI-1640 medium containing 10\% fetal bovine serum; GE Healthcare Life Sciences) after $24 \mathrm{~h}$. The expression of SPC25 shRNA in infected cells was determined by reverse transcription-quantitative polymerase chain reaction (RT-qPCR). The SPC25 shRNA sequence was as follows: 3'-CCGGCCATCA AAGCATTTGCAGAAACTCGAGTTTCTGCAAATGCTT TGATGGTTTTTG-5', which were purchased from Shanghai GenePharma Co., Ltd. (Shanghai, China). Knockdown effects of these shRNA plasmids on endogenous SPC25 expression were validated $48 \mathrm{~h}$ following their transfection using by RT-qPCR.

Cell culture. Prostate cancer PC-3 cells and 293T cells were purchased from the American Type Culture Collection (Manassas, VA, USA) and confirmed by short tandem repeat analysis (20). Cells were cultured in RPMI-1640 medium containing $10 \%$ fetal bovine serum (GE Healthcare Life
Sciences), $100 \mathrm{U} / \mathrm{ml}$ penicillin and $100 \mu \mathrm{g} / \mathrm{ml}$ streptomycin, and were cultured at $37^{\circ} \mathrm{C}$ in $5 \% \mathrm{CO}_{2}$.

$R T-q P C R$. Total RNA was extracted from PC-3 cells using TRIzol reagent (Invitrogen; Thermo Fisher Scientific, Inc.). cDNA was synthesized using a PrimeScript RT reagent kit (Takara Biotechnology Co., Ltd., Dalian, China). qPCR was performed with cDNA samples using the iQSYBRGreenSupermix and ABI Prism 7900 platform (both Bio-Rad Laboratories, Inc., Hercules, CA, USA), according to the manufacturer's protocol. PCR cycling conditions were $50^{\circ} \mathrm{C}$ for $2 \mathrm{~min}$, followed by $95^{\circ} \mathrm{C}$ for $10 \mathrm{~min}, 40$ cycles of $95^{\circ} \mathrm{C}$ for $15 \mathrm{sec}$ and $60^{\circ} \mathrm{C}$ for $1 \mathrm{~min}$. The $2^{-\Delta \Delta \mathrm{Cq}}$ method was used to calculate the relative expression level by normalizing to GAPDH levels. The following primer sequences were used: SPC25 forward, 5'-AGAAGAACGAATGGTTGAGAT-3' and reverse, 5'-TCCTGGATATTTGCAGTCAGT-3'; and GAPDH forward, 5'-TGACTTCAACAGCGACACCCA-3' and reverse, 5'-CACCCTGTTGCTGTAGCCAAA-3'. Each sample was run in triplicate to ensure quantitative accuracy.

Plate analysis with the adherent cell cytometry system Celigo ${ }^{\circledR}$. PC-3 cells were stained with fluorescence nuclear staining (Hoechst nuclei stain; $2.6 \mu \mathrm{g} / \mathrm{ml}$; Invitrogen; Thermo Fisher Scientific, Inc.) for $10 \mathrm{~min}$ at $37^{\circ} \mathrm{C}$. The adherent cell cytometry system, Celigo ${ }^{\circledR}$ (analyzed by Application Programing Interface, version 1.0, software), allowed rapid quantification of cellular fluorescence expression as previously described (21). siGLO Green Transfection Indicator (50 nM, Dharmacon Inc., Lafayette, CO, USA) was transfected into PC-3cells with DharmaFECT 1 (0.15 $\mu \mathrm{g} / 100 \mu \mathrm{l}$ well, Dharmacon Inc.). After $24 \mathrm{~h}$, cells were washed with 1 X PBS and stained with Hoechst nuclei stain $(2.6 \mu \mathrm{g} / \mathrm{ml}$; Invitrogen; Thermo Fisher Scientific, Inc.). Plates were analyzed using the adherent cell cytometer equipped with bright field and fluorescent channels: A blue 4',6-diamidino-2-phenylindole filter for the Hoechst nuclei stain and a green filter for the siGLOGreen (Dharmacon Inc.). Gating parameters were adjusted for each fluorescence channel to exclude background and other non-specific signals. The Celigo ${ }^{\circledR}$ system provided a gross quantitative analysis for each fluorescence channel and individual well, including total count and average integrated red fluorescence intensity of gated events.

Cell proliferation assay. An MTT assay was performed in order to evaluate changes in cell proliferation. A total of 5,000 transfected PC-3 cells were seeded onto 96-well plates at a final volume of $100 \mu \mathrm{l}$ medium/well (RPMI-1640 medium containing $10 \%$ fetal bovine serum; GE Healthcare Life Sciences). Proliferation was assessed at 0, 24, 48, 72 and 96 h. Cell proliferation was quantified by adding $20 \mu \mathrm{l}$ MTT $(0.5 \mathrm{mg} / \mathrm{ml}$; Sigma-Aldrich; Merck KGaA, dissolved in dimethyl sulfoxide; Sigma-Aldrich; Merck KGaA), according to the manufacturer's protocol. Following a $4 \mathrm{~h}$ incubation, the plates were monitored using a PowerWave XS Microplate reader (BioTek Instruments, Inc., Winooski, VT, USA), which measured absorbance at $490 \mathrm{~nm}$. The absorbance at $570 \mathrm{~nm}$ was used as a reference. Each experiment was performed at least in triplicate.

Cell cycle and apoptosis assay. Following transfection for $48 \mathrm{~h}$, cells were harvested and washed with phosphate-buffered saline 
A
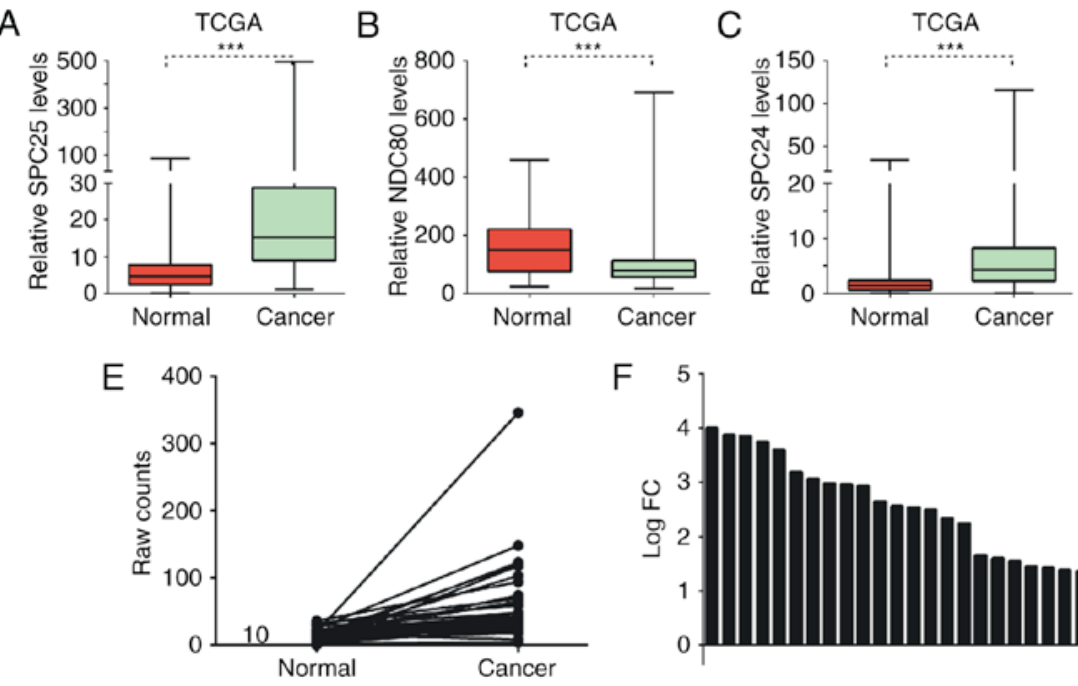

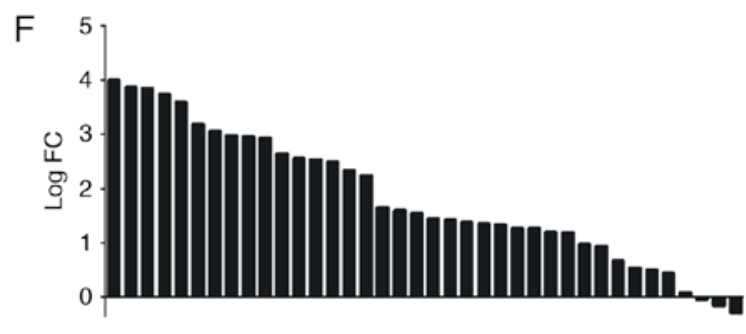

Figure 1. SPC25 expression was upregulated in PCa samples. TCGA data analysis demonstrated that (A) SPC25, (B) NDC80, (C) SPC24 and (D) NUF2 were differentially expressed in PCa samples compared with normal prostate tissues. (E) SPC25 was significantly upregulated in PCa samples compared with matched normal samples. (F) In the TCGA cohorts, $>90 \%$ (35/38) of the paired PCa cases exhibited positive fold changes in SPC25 expression between the PCa samples and the adjacent normal tissues. $\mathrm{P}<0.05$ was considered to indicate a statistically significant difference. ${ }^{* * *} \mathrm{P}<0.001$. SPC 25 , spindle pole body component 25 homolog; PCa, prostate cancer; TCGA, The Cancer Genome Atlas.

(PBS) three times. Cells were incubated with PBS containing $0.03 \%$ Triton X-100, $100 \mathrm{ng} / \mathrm{ml}$ RNase A and $50 \mathrm{ng} / \mathrm{ml}$ propidium iodide (PI) for $15 \mathrm{~min}$ at $37^{\circ} \mathrm{C}$. The distribution of cells in the different phases of the cell cycle was analyzed using a FACSanto flow cytometer (BD Biosciences, San Jose, CA, USA). Data on cell cycle distribution were analyzed using ModFit LT 3.0 software (BD Biosciences). For the apoptosis assay, cells were assayed with an Annexin V-APC Apoptosis Detection kit (eBioscience; Thermo Fisher Scientific, Inc.) and were analyzed using a flow cytometer (BD Biosciences).

Microarray and expression datasets. Total RNA was extracted from shSPC25 and shRNA control samples using TRIzol reagent (Invitrogen; Thermo Fisher Scientific, Inc.) and the RNeasy mini kit (Qiagen GmbH, Hilden, Germany). Total RNA was quantified by the NanoDrop ND-2000 (Thermo Fisher Scientific, Inc.) and the RNA integrity was assessed using Agilent Bioanalyzer 2100 (Agilent Technologies, Inc., Santa Clara, CA, USA).

Global expressions of mRNAs in 3 SPC25 shCtrl samples and 3 shSPC25 were examined using the Gene Chip Prime View Human Gene Expression Array (Thermo Fisher Scientific, Inc.). The sample labeling, microarray hybridization and washing were performed according to the manufacturer's protocols. The raw microarray data were uploaded to the Gene Expression Omnibus (GEO) public repository (http://www. ncbi.nlm.nih.gov/geo/; GEO series no. GSE73397). Raw data were normalized using the $\log 2$ scale. Two-class unpaired significance analysis of microarray (22) was employed to filter significantly differentially expressed mRNAs between shCtrl and shSPC25. To begin with, the raw data were normalized using the quantile algorithm. The probes for which $\geq 1$ out of 2 conditions have flags in ' $\mathrm{P}$ ' were selected for further data analysis. Differentially expressed mRNAs were subsequently identified through fold change. The threshold set for upregulated and downregulated genes was a fold change $\geq 2$.0. Subsequently, Kyoto Encyclopedia of Genes and Genomes (KEGG) pathway and Gene Ontology (GO) analyses of differently expressed downstream genes of SPC25 were performed using an Ingenuity Pathway Analysis system (https://www.qiagenbioinformatics. com/products/ingenuity-pathway-analysis/).

Statistical analysis. The numerical data are presented as the mean \pm standard deviation of $\geq 3$ repeats. To determine the associations between SPC25 expression and the clinical characteristics of the tumors, Student's t-tests or Mann-Whitney U-tests were used as appropriate. For variables $\geq 3$ groups, the Kruskal-Wallis $\mathrm{H}$ test was used for non-parametric analysis. Tukey HSD was used as the post hoc test. Kaplan Meier analysis, followed by the log-rank test, and Cox regression analyses were utilized to assess the association between SPC25 and overall survival as well as the prognosis of PCa. All tests were two-sided and $\mathrm{P}<0.05$ was considered to indicate a statistically significant difference. Statistical analysis was performed using the SPSS software package, version 15.0 (SPSS, Inc., Chicago, IL, USA).

\section{Results}

SPC25 was upregulated in PCa. The present study used the TCGA database to investigate the expression pattern of the Ndc80 complex in PCa. It was revealed that SPC25, SPC24 and NUF2 were significantly upregulated and NDC80 was downregulated in PCa samples compared with matched normal tissues (Fig. 1A-D). To the best of our knowledge, the functional roles of SPC25 in cancer had not previously been reported. Therefore, SPC25 was selected for further study. The results of the present study demonstrated that SPC 25 was significantly upregulated in PCa samples compared with matched normal tissues (Fig. 1E). Additionally, $>90 \%$ of PCa tissues expressed high levels of SPC25, while only $10 \%$ (3/38) of matched adjacent normal tissues expressed high levels of SPC25 (Fig. 1F).

SPC25 knockdown inhibits cell proliferation in PC-3 cells. In order to characterize the role of SPC25 in the PC-3 cell line, SPC25 shRNA was used to knockdown its expression. 


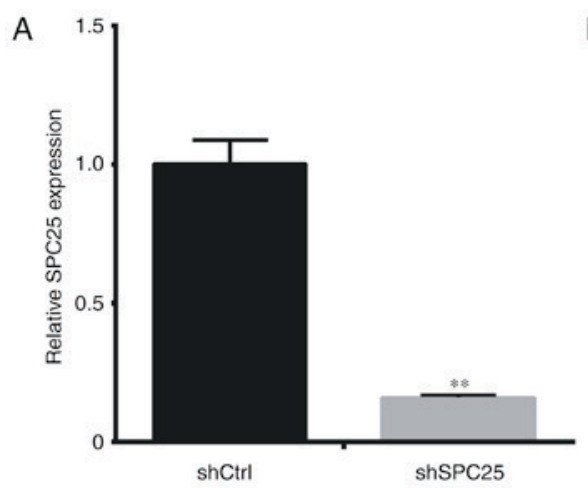

B

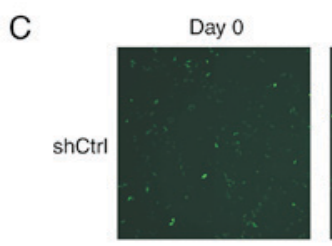

Day 1
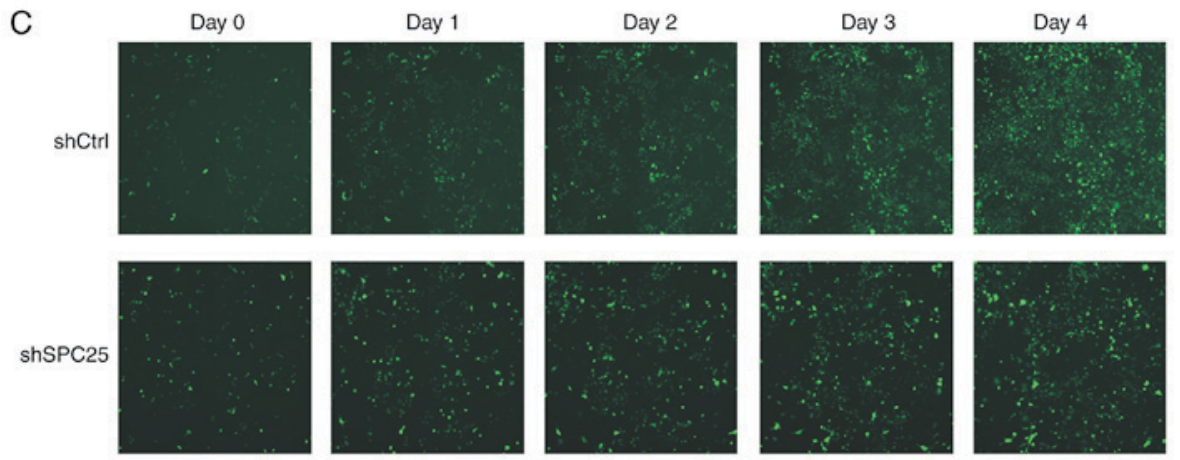

$\mathrm{D}$

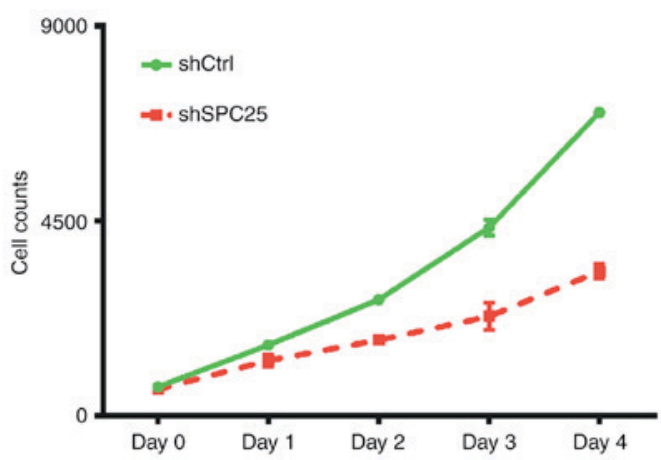

Figure 2. SPC25 knockdown inhibits the proliferation of PC-3 cells. (A) Expression of SPC25 mRNA following transfection with the indicated shRNAs in PC-3 cells. (B) An MTT assay demonstrated that knockdown of SPC25 inhibited cell proliferation in PC-3 cells. (C) The Celigo ${ }^{\circledR}$ system revealed that knockdown of SPC25 inhibited cell proliferation in PC-3 cells. (D) Cell number of shCtrl or shSPC25 group in each day was calculated using the Celigo ${ }^{\circledR}$ system. $\mathrm{P}<0.05$ was considered to indicate a statistically significant difference. ${ }^{* *} \mathrm{P}<0.01 ;{ }^{* * * *} \mathrm{P}<0.001$. SPC25, spindle pole body component 25 homolog; sh, short hairpin; Ctrl, control; OD, optical density.

The results of the present study demonstrated that shSPC25 significantly reduced SPC25 mRNA expression compared with expression in cells transfected with the negative control (Fig. 2A). When PC-3cells were transfected with shSPC25, cell proliferation was decreased (Fig. 2B-D).

SPC25 knockdown decreased the number of PCa cells in the $S$ phase and increased the number in the G2/M phase. Subsequently, the effect of SPC25 knockdown on cell cycle progression in PC-3 cells was determined using a flow cytometer. Knockdown of SPC25 in PC-3 cells increased the percentage of cells in the G2/M phase and decreased the percentage of cells in the $\mathrm{S}$ phase, but did not affect the percentage of cells in the G1 phase compared with cells treated with the negative control (Fig. 3A and B). These results indicated that SPC25 was involved in regulating the PCa cell cycle.

SPC25 knockdown promotes the apoptosis of PCa cells. Subsequently, the role of SPC25 in the apoptosis of PCa cells was investigated. PC-3 cells stably expressing shSPC25 and stained with Annexin V-APC were analyzed by flow cytometry. As demonstrated in Fig. 4, SPC25 knockdown promoted the apoptosis of PC-3 cells.

Bioinformatics analysis reveals multiple functional roles of SPC25 in PCa. To further investigate the functional roles of SPC25 in PCa, mRNA expression profiling was used to detect global gene expression levels following SPC25 knockdown. Analysis of the microarray data revealed 193 upregulated and 297 downregulated genes following SPC25 knockdown, with an average expression level $>1.5$-fold $(\mathrm{P}<0.05$; Fig. 5A). Subsequently, Kyoto Encyclopedia of Genes and Genomes (KEGG) pathway and Gene Ontology (GO) analyses of differently expressed downstream genes of SPC25 were performed using an Ingenuity Pathway Analysis system (www.ingenuity.com). SPC25 was significantly involved in regulating role of tissue factor in cancer, mouse embryonic stem cell pluripotency pathway, transforming growth factor- $\beta$ 

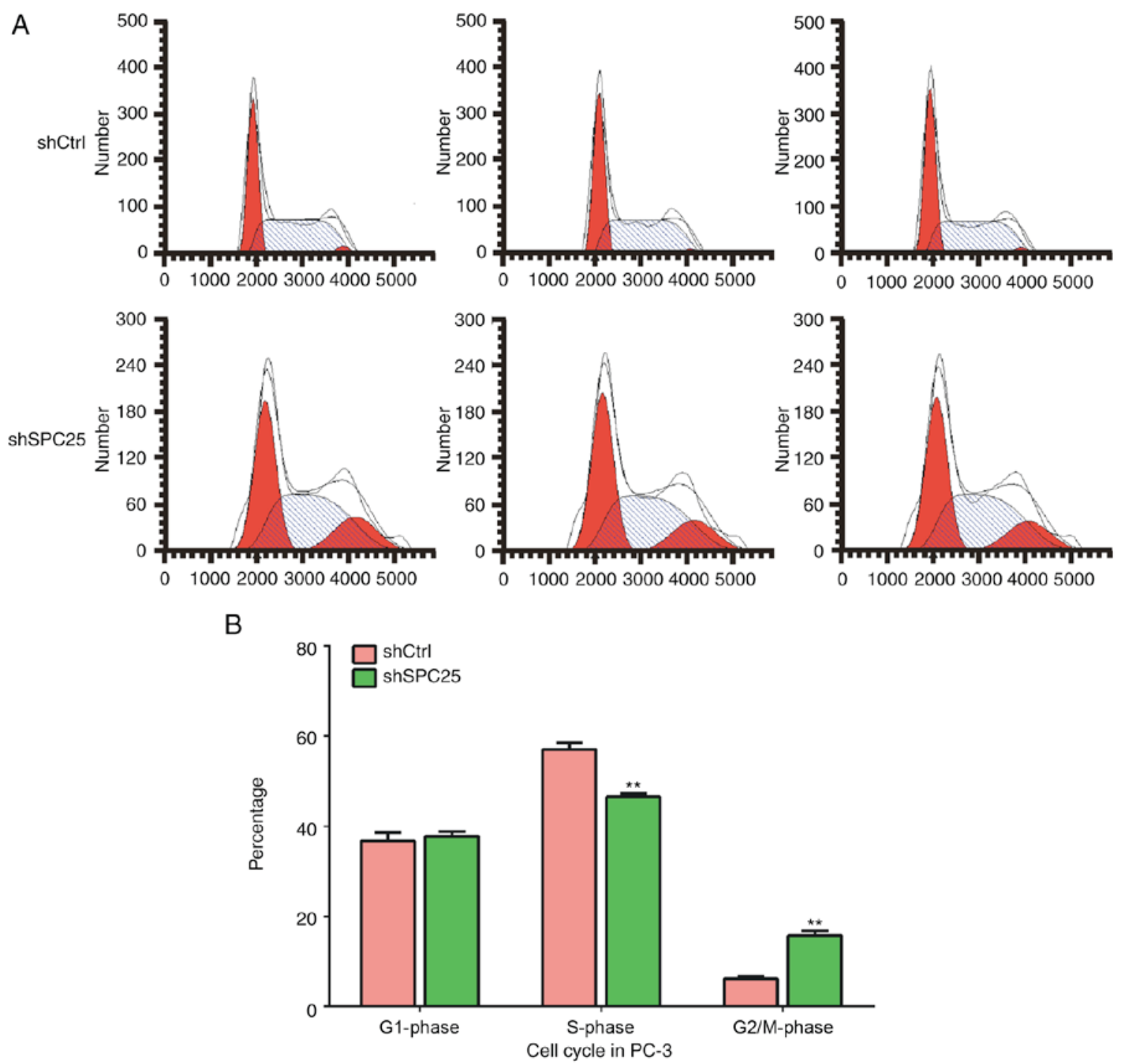

Figure 3. SPC25 knockdown induced a decrease in the number of PCa cells in the S phase and an increase in the number of PCa cells in the G2/M phase. (A) The shSPC25-induced decrease in the number of PCa cells in the S phase and increase in the number of PCa cells in the G2/M phase. (B) Quantitative representation of the shSPC25-induced decrease in the number of PCa cells in the S phase and increase in the number of PCa cells in the G2/M phase. The cell cycle analysis results are presented as the mean \pm standard deviation $(n=3)$. $\mathrm{P}<0.05$ was considered to indicate a statistically significant difference. ${ }^{* * *} \mathrm{P}<0.01$ vs. shCtrl. SPC25, spindle pole body component 25 homolog; PCa, prostate cancer; sh, short hairpin.

signaling, SUMOylation pathway, retinoic acid receptor activation, extracellular-signal-regulated kinase 5 signaling, bladder cancer signaling, hepatocyte growth factor signaling and nerve growth factor signaling (Fig. 5B). GO analysis demonstrated that SPC25 widely participated in the regulation of the migration of cells, cell movement, cell proliferation of tumor cell lines, angiogenesis, development of vasculature, vasculogenesis, growth of tumor, cell movement of tumor cells, enlargement of cells, organismal death, morbidity or mortality, apoptosis, growth of epithelial tissue and invasion of cells (Fig. 5C).

Upregulation of SPC25 predicts a poor prognosis in PCa. Furthermore, the present study evaluated the possible prognostic value of SPC25 using TCGA RNA-seq data. The results demonstrated that the expression level of SPC25 was significantly associated with the pathological $\mathrm{T}$ stage and the $\mathrm{N}$ stage of PCa. The results of the present study revealed that SPC25 levels were higher in N1 stage PCa samples than
inN0 stage PCa samples (Fig. 6A). It was also revealed that SPC25 expression was significantly upregulated in T3/T4 PCa samples compared with expression in T2 PCa samples (Fig. 6B). These results suggested that SPC25 may be involved in PCa metastasis.

Analysis of the TCGA database demonstrated that a significantly higher expression of SPC25 was observed in Gleason 8 $(\mathrm{P}<0.001)$ and Gleason $9(\mathrm{P}<0.001)$ patients compared with Gleason 6 and Gleason 7 patients (Fig. 6C) (19).

In order to further investigate the prognostic value of SPC25 in PCa, the overall survival rates of patients with high or low SPC25 expression were assessed using the Kaplan-Meier method. Using the Taylor dataset (GSE21032) (23), 75\% SPC25 mRNA expression in all PCa tissues was used as the cut-off point to divide all cases into high $(n=36)$ and low $(n=105)$ SPC25 expression groups. As demonstrated in Fig. 6D, compared with patients with high SPC25 expression, the 5 -year BCR-free survival rates were higher in patients with low SPC25 expression in this dataset (Fig. 6D), indicating that 
A
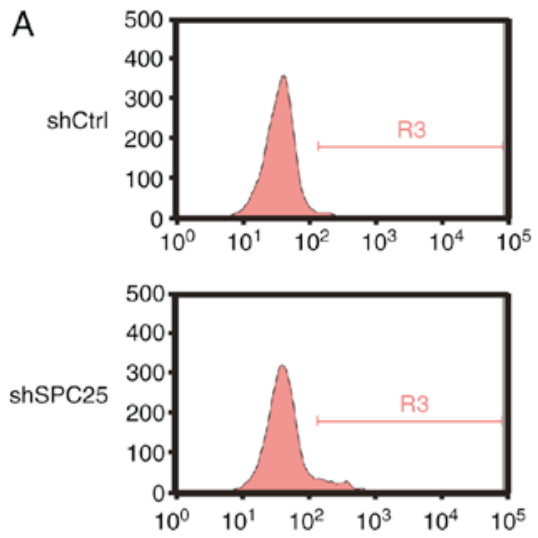
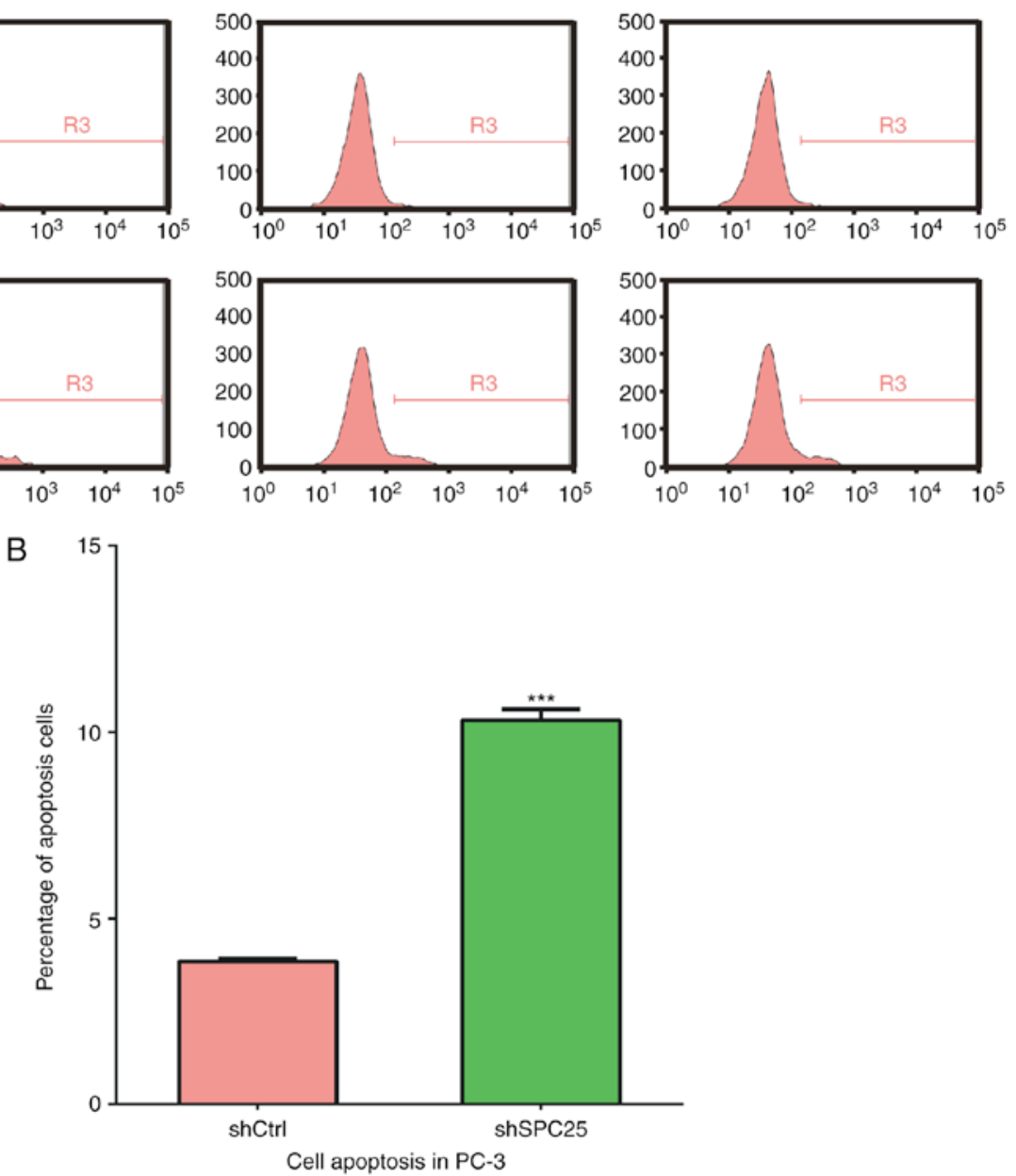

Figure 4. SPC25 knockdown promotes apoptosis of prostate cancer cells. (A) Flow cytometric analysis of the shSPC25-induced apoptosis of PC-3 cells (B) Quantitative representation of the shSPC25-induced apoptosis of PC-3 cells. The cell apoptosis analysis results are presented as the mean \pm standard deviation $(\mathrm{n}=3)$. $\mathrm{P}<0.05$ was considered to indicated a statistically significant difference. ${ }^{* * *} \mathrm{P}<0.001 \mathrm{vs}$. shCtrl. SPC25, spindle pole body component 25 homolog; sh, short hairpin; Ctrl, control.

the low level of SPC25 was associated with a longer BCR-free survival time.

\section{Discussion}

SPC25 is a member of the Ndc80 complex that serves an important role in regulating mitotic chromosome segregation (4). Abnormal expression of the Ndc80 complex was observed in different types of human cancer (5-7). For example, SPC24, a co-factor of SPC25, was revealed to be critical for the progression of anaplastic thyroid cancer (24). The present study focused on investigating the roles of SPC25 in PCa. In a previous study, SPC25 was significantly overexpressed in human breast tumor tissues and was associated with reduced overall survival (25). However, the functional roles of SPC25 in cancer remain unknown.

The present study analyzed the TCGA database in order to investigate the expression pattern of the Ndc80 complex in PCa. The results demonstrated that SPC25 was significantly upregulated in PCa samples compared with expression in matched normal tissues. These results suggested that SPC25 may act as an oncogene in PCa. In order to characterize the role of SPC25 in PCa cells, a loss of function assay was performed in the present study. The results demonstrated that SPC25 knockdown may significantly reduce PCa cell proliferation. Given that SPC25 is an important component of the mitotic checkpoint machinery, the roles of SPC25 in regulating the PCa cell cycle were also investigated. It was revealed that SPC25 knockdown induced a decrease in the number of PCa cells in the S phase and an increase in the number of PCa cells in the G2/M phase. Furthermore, SPC25 knockdown promoted the apoptosis of $\mathrm{PCa}$ cells. Taken together, these results demonstrated that SPC25 serves an oncogenic role in PCa by regulating the cell cycle and apoptosis.

The present study demonstrated the effect of SPC25 on cell cycle regulation. In order to further investigate the functional roles of SPC25 in PCa, bioinformatics analysis was performed, in combination with a high-throughput array. A total of 193 genes were identified to be upregulated and 297 genes were identified to be downregulated following SPC25 knockdown. In line with the experimental results of the present study, GO analysis revealed that SPC25 is involved in regulating cell proliferation and apoptosis. Notably, SPC25 downstream genes were revealed to be significantly enriched in cell invasion pathways. Furthermore, KEGG pathway analysis demonstrated that SPC25 was involved in regulating role of tissue factor in cancer, mouse embryonic stem cell pluripotency, transforming growth factor- $\beta$ signaling and SUMOylation 
A

KD1 KD3 KD2 NC1 NC2 NC3
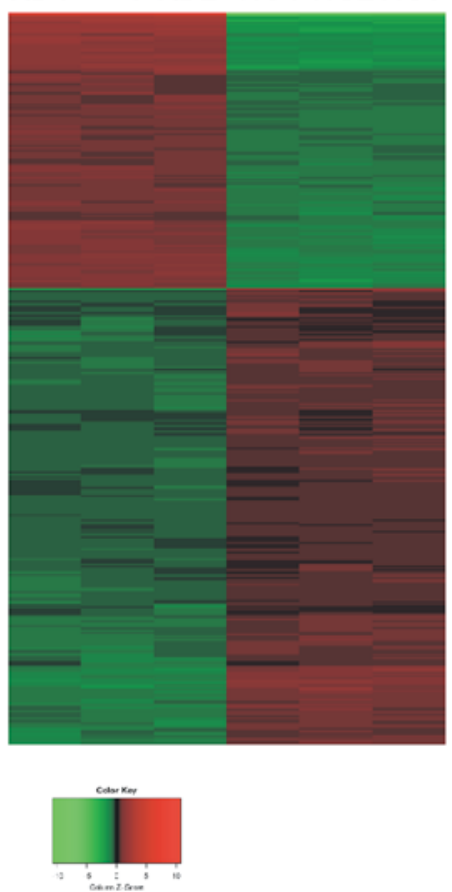

B

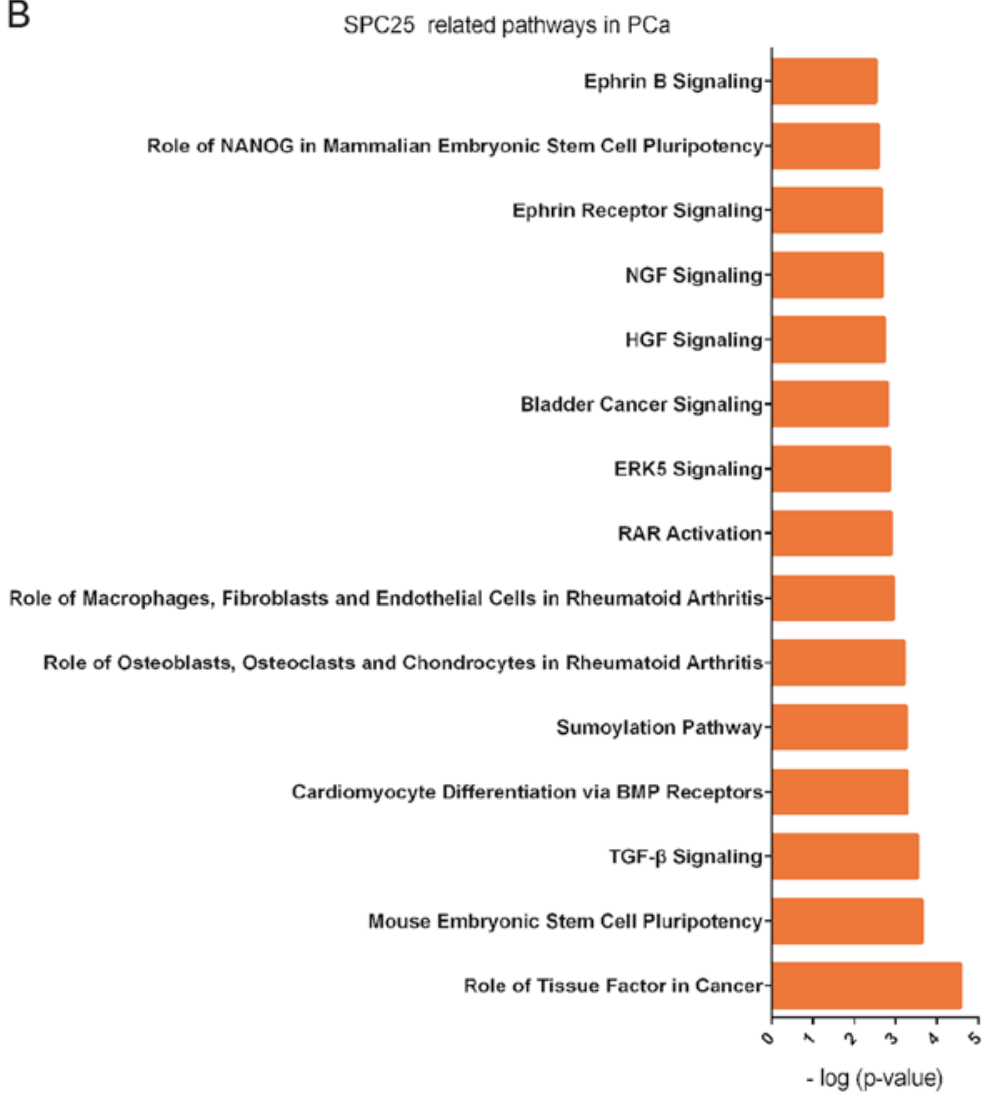

C

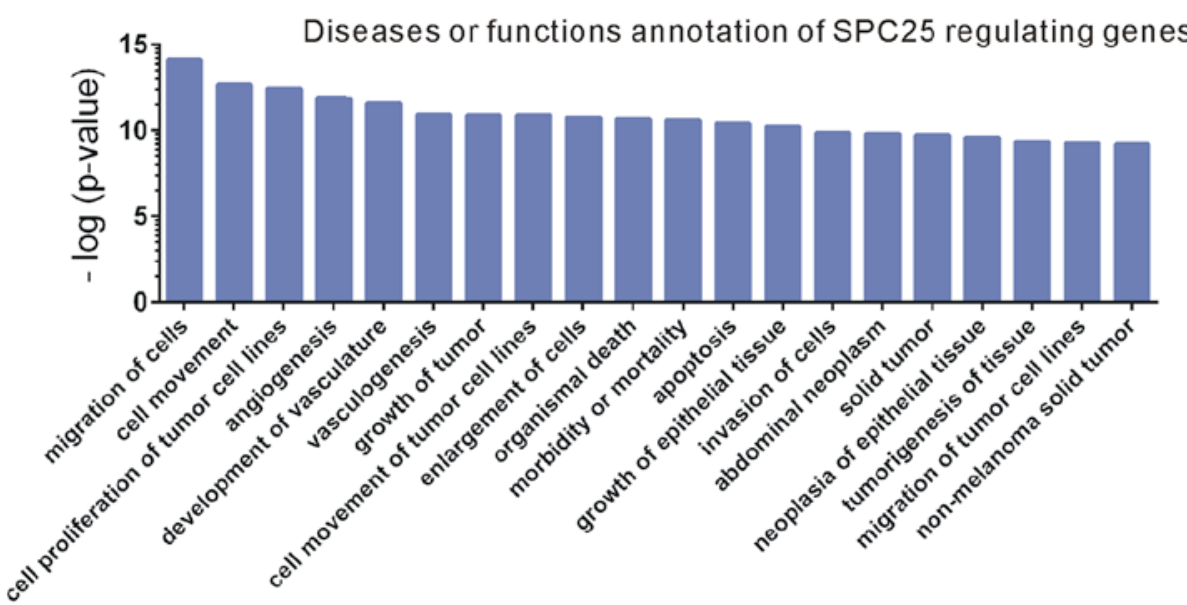

Figure 5. Bioinformatics analysis revealed multiple functional roles of SPC25 in PCa. (A) Heat map demonstrates differential genes expression following SPC25 knockdown. (B) IPA analysis demonstrated the potential pathways regulated by SPC25. (C) IPA analysis demonstrated the potential diseases and functions effected by SPC25 knockdown in PCa. SPC25, spindle pole body component 25 homolog; PCa, prostate cancer; IPA, ingenuity pathway analysis; KD, knockdown; NC, negative control.

pathway. Although further validation is required, the results of the present study provided novel information regarding the role of SPC25 in regulating PCa progression.

$\mathrm{PCa}$ is one of the most frequently diagnosed types of cancer worldwide (1). In the last three decades, a series of genes, including prostate-specific antigen (PSA) $(26,27)$, prostate cancer associated 3 (26), and ubiquitin-like with PHD and RING finger domains (28) were revealed to be dysregulated in PCa and thus, may act as biomarkers. Notably, PSA testing was the most widely used biomarker of PCa (27). However, there are limitations regarding the accuracy of these tests (29). Therefore, there remains an urgent requirement to identify novel biomarkers for PCa. In the present study, the possible prognostic value of SPC25 was evaluated using TCGA RNA-seq data. According to this analysis, the expression level of SPC25 was significantly upregulated in T3/T4 PCa samples compared with T2 PCa samples. These results also revealed that SPC25 levels were higher in N1 stage PCa samples than in N0 stage PCa samples. Analysis of the TCGA database also demonstrated that a significantly higher expression of SPC25 was observed in patients with high Gleason scores (Gleason 8 and Gleason 9) than in patients with low Gleason scores (Gleason 6 and Gleason 7). Kaplan-Meier analysis revealed that patients with PCa with a low expression of SPC 25 had a longer 
A

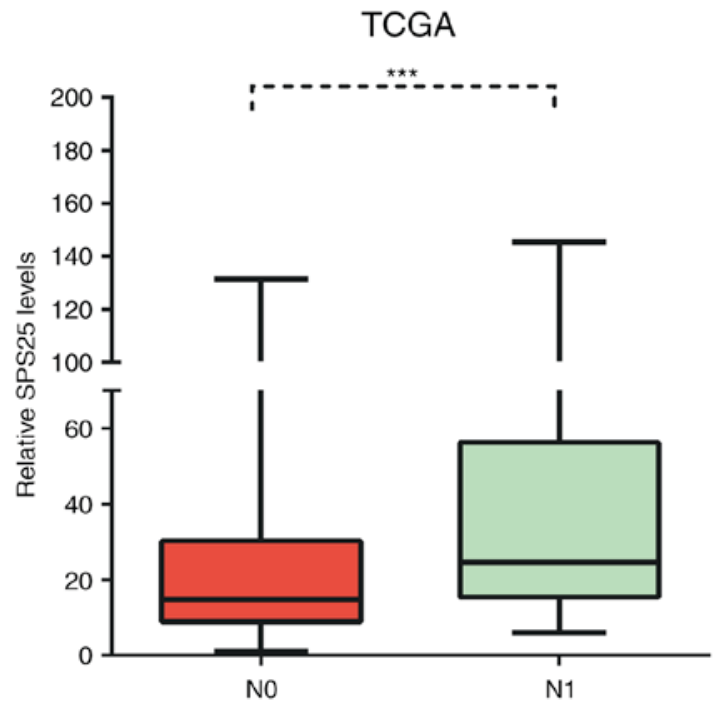

C

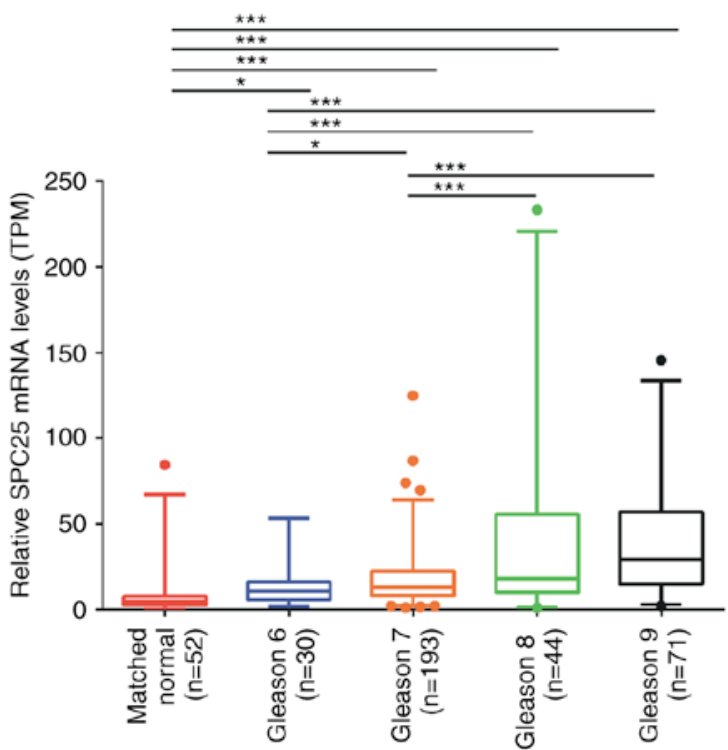

B

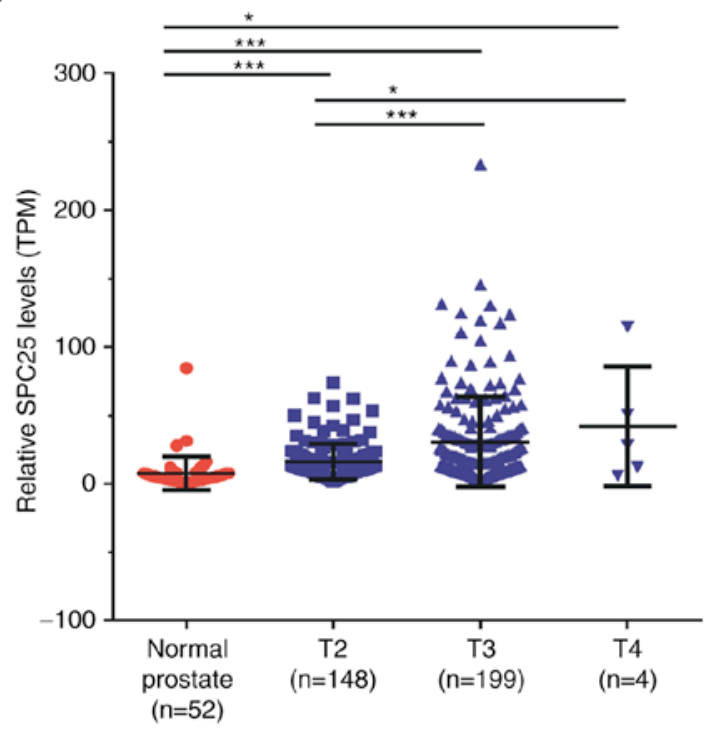

D

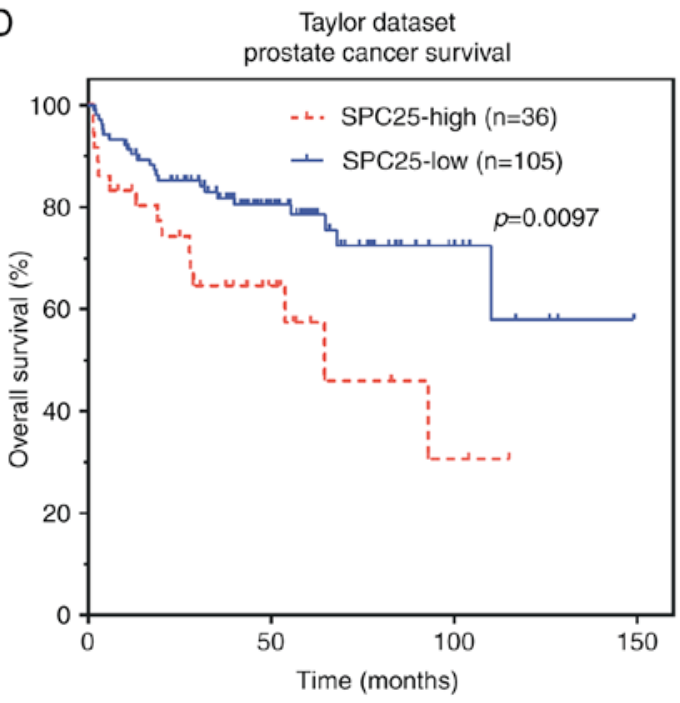

Figure 6. Upregulation of SPC25 predicts a poor prognosis in PCa. (A) SPC25 expression was upregulated in N1 PCa cases compared with N0 PCa cases. (B) SPC25 expression was upregulated in T3/T4 PCa cases compared with T2 PCa cases. (C) SPC25 expression was upregulated in Gleason 8 and Gleason 9 PCa cases compared with Gleason 6 or Gleason 7 PCa cases. (D) Compared with patients exhibiting a high SPC25 expression, the overall survival rates were higher in patients exhibiting a low SPC 25 expression in the TCGA database. $\mathrm{P}<0.05$ was considered to indicate a statistically significant difference. ${ }^{*} \mathrm{P}<0.05$; ${ }^{* * *} \mathrm{P}<0.001$. SPC25, spindle pole body component 25 homolog; TPM, Transcripts Per Kilobase Million; PCa, prostate cancer; TCGA, The Cancer Genome Atlas.

BCR-free survival time than those with a high expression of SPC25. To the best of our knowledge, the present study was the first to report that SPC25 was involved in the prognosis of PCa.

To the best of our knowledge, the present study was the first to demonstrate that SPC25 was significantly upregulated in PCa. In order to investigate the molecular functional roles of SPC25, a loss of function assay was performed and it was revealed that SPC25 knockdown inhibited cell proliferation and induced a decrease in the number of $\mathrm{PCa}$ cells in the $\mathrm{S}$ phase and an increase in the number of cells in the G2/M phase. Further more, SPC25 knockdown promoted the apoptosis of PCa. Notably, bioinformatics analysis revealed multiple functional roles of SPC25 in regulating cell proliferation, apoptosis, invasion, role of tissue factor in cancer, transforming growth factor- $\beta$ signaling, and SUMOylation pathway in PCa. Furthermore, the present study evaluated the possible prognostic value of SPC25 using TCGA
RNA-seq data and revealed that SPC25 was upregulated in late pathological stages of PCa. Kaplan-Meier analysis demonstrated that lower SPC25 expression levels were associated with better survival of patients with PCa. Taken together, the results of the present study suggested that SPC25 serves an oncogenic role in $\mathrm{PCa}$ and may act as a novel diagnostic and therapeutic target for PCa.

\section{Acknowledgements}

Not applicable.

\section{Funding}

The present study was supported by the Social Development Plan of Jiangsu Province-Standardization of Key Disease 
Diagnosis and Treatment Projects (grant no. BE2016715) from Jiangsu Province Science and Technology Commission.

\section{Availability of data and materials}

The datasets used and/or analyzed during the current study are available from the corresponding author on reasonable request.

\section{Author's contributions}

FC and HT were responsible for the study conception and design. FC, JH, YF and HT developed the methodology. FC, YF and JT analysed and interpreted the data. FC and HT wrote, reviewed and revised the manuscript.

\section{Ethics approval and consent to participate}

Not applicable.

\section{Consent for publication}

Not applicable.

\section{Competing interests}

The authors declare that they have no competing interests.

\section{References}

1. Dominguez-Brauer C, Thu KL, Mason JM, Blaser H, Bray MR and Mak TW: Targeting mitosis in cancer: Emerging strategies. Mol Cell 60: 524-536, 2015.

2. DeLuca JG, Gall WE, Ciferri C, Cimini D, Musacchio A and Salmon ED: Kinetochore microtubule dynamics and attachment stability are regulated by Hec1. Cell 127: 969-982, 2006.

3. Ciferri C, Pasqualato S, Screpanti E, Varetti G, Santaguida S, Dos Reis G, Maiolica A, Polka J, De Luca JG, De Wulf P, et al: Implications for kinetochore-microtubule attachment from the structure of an engineered Ndc80 complex. Cell 133: 427-439, 2008.

4. Tooley J and Stukenberg PT: The Ndc80 complex: Integrating the kinetochore's many movements. Chromosome Res 19: 377-391, 2011

5. Hu P, Chen X, Sun J, Bie P and Zhang LD: siRNA-mediated knockdown against NUF2 suppresses pancreatic cancer proliferation in vitro and in vivo. Biosci Rep 35: pii: e00170, 2015.

6. Fu HL and Shao L: Silencing of NUF2 inhibits proliferation of human osteosarcoma Saos-2 cells. Eur Rev Med Pharmacol Sci 20: 1071-1079, 2016.

7. Xing XK, Wu HY, Chen HL and Feng HG: NDC80 promotes proliferation and metastasis of colon cancer cells. Genet Mol Res 15, 2016

8. Suzuki A, Badger BL, Haase J, Ohashi T, Erickson HP, Salmon ED and Bloom K: How the kinetochore couples microtubule force and centromere stretch to move chromosomes. Nat Cell Biol 18: 382-392, 2016.

9. Wan X, Huang W, Yang S, Zhang Y, Pu H, Fu F, Huang Y, Wu H, $\mathrm{Li} \mathrm{T}$ and $\mathrm{Li} \mathrm{Y}$ : Identification of androgen-responsive lncRNAs as diagnostic and prognostic markers for prostate cancer. Oncotarget 7 : 60503-60518, 2016.

10. Chang KH, Li R, Kuri B, Lotan Y, Roehrborn CG, Liu J, Vessella R, Nelson PS, Kapur P, Guo X, et al: A gain-of-function mutation in DHT synthesis in castration-resistant prostate cancer. Cell 154: 1074-1084, 2013

11. Zhang P, Gao K, Tang Y, Jin X, An J, Yu H, Wang H, Zhang Y, Wang D, Huang H, et al: Destruction of DDIT3/CHOP protein by wild-type SPOP but not prostate cancer-associated mutants. Hum Mutat 35: 1142-1151, 2014.
12. An J, Ren S, Murphy SJ, Dalangood S, Chang C, Pang X, Cui Y, Wang L, Pan Y, Zhang X, et al: Truncated ERG oncoproteins from TMPRSS2-ERG fusions Are resistant to SPOP-mediated proteasome degradation. Mol Cell 59: 904-916, 2015.

13. Zhang L, Wang J, Wang Y, Zhang Y, Castro P, Shao L, Sreekumar A, Putluri N, Guha N, Deepak S, et al: MNX1 Is oncogenically upregulated in African-American prostate cancer. Cancer Res 76: 6290-6298, 2016.

14. Das M: MNX1: A novel prostate cancer oncogene. Lancet Oncol 17: e521, 2016.

15. Xie JJ, Zhuo YJ, Zheng Y, Mo RJ, Liu ZZ, Li BW, Cai ZD, Zhu XJ, Liang YX, He HC and Zhong WD: High expression of ASPM correlates with tumor progression and predicts poor outcome in patients with prostate cancer. Int Urol Nephrol 49: 817-823, 2017.

16. Schlomm T: Re: The molecular taxonomy of primary prostate cancer. Eur Urol 69: 1157, 2016.

17. Cancer Genome Atlas Research Network: The molecular taxonomy of primary prostate cancer. Cell 163: 1011-1025, 2015.

18. Buyyounouski MK, Choyke PL, McKenney JK, Sartor O, Sandler HM, Amin MB, Kattan MW and Lin DW: Prostate cancer-major changes in the American Joint Committee on Cancer eighth edition cancer staging manual. CA Cancer J Clin 67: 245-253, 2017.

19. Rees MA, Resnick MI and Oesterling JE: Use of prostate-specific antigen, Gleason score, and digital rectal examination in staging patients with newly diagnosed prostate cancer. Urol Clin North Am 24: 379-388, 1997.

20. Dirks WG and Drexler HG: STR DNA typing of human cell lines: Detection of intra- and interspecies cross-contamination. Methods Mol Biol 946: 27-38, 2013.

21. Nabzdyk CS, Chun M, Pradhan Nabzdyk L, Yoshida S and LoGerfo FW: Differential susceptibility of human primary aortic and coronary artery vascular cells to RNA interference. Biochem Biophys Res Commun 425: 261-265, 2012.

22. Tusher VG, Tibshirani R and Chu G: Significance analysis of microarrays applied to the ionizing radiation response. Proc Natl Acad Sci USA 98: 5116-5121, 2001.

23. Taylor BS, Schultz N, Hieronymus H, Gopalan A, Xiao Y, Carver BS, Arora VK, Kaushik P, Cerami E, Reva B, et al: Integrative genomic profiling of human prostate cancer. Cancer Cell 18: 11-22, 2010.

24. Yin H, Meng T, Zhou L, Chen H and Song D: SPC24 is critical for anaplastic thyroid cancer progression. Oncotarget 8: 21884-21891, 2017.

25. Pathania R, Ramachandran S, Mariappan G, Thakur P, Shi H, Choi JH, Manicassamy S, Kolhe R, Prasad PD, Sharma S, et al: Combined Inhibition of DNMT and HDAC blocks the tumorigenicity of cancer stem-like cells and attenuates mammary tumor growth. Cancer Res 76: 3224-3235, 2016.

26. Tsaur I, Hennenlotter J, Oppermann E, Munz M, Kuehs U, Stenzl A and Schilling D: PCA3 and PSA gene activity correlates with the true tumor cell burden in prostate cancer lymph node metastases. Cancer Biomark 15: 311-316, 2015.

27. Prensner JR, Rubin MA, Wei JT and Chinnaiyan AM: Beyond PSA: The next generation of prostate cancer biomarkers. Sci Transl Med 4: 127rv3, 2012.

28. Wan X, Yang S, Huang W, Wu D, Chen H, Wu M, Li J, Li T and Li Y: UHRF1 overexpression is involved in cell proliferation and biochemical recurrence in prostate cancer after radical prostatectomy. J Exp Clin Canc Res 35: 34, 2016.

29. D'Amico AV, Whittington R, Malkowicz SB, Wu YH, Chen M, Art M, Tomaszewski JE and Wein A: Combination of the preoperative PSA level, biopsy gleason score, percentage of positive biopsies, and MRI T-stage to predict early PSA failure in men with clinically localized prostate cancer. Urology 55: 572-577, 2000 .

This work is licensed under a Creative Commons Attribution-NonCommercial-NoDerivatives 4.0 International (CC BY-NC-ND 4.0) License. 